\title{
Quantum tops as examples of commuting differential operators
}

\author{
V.E. Adler, V.G. Marikhin, A.B. Shabat
}

26 September 2011

\begin{abstract}
We study the quantum analogs of tops on Lie algebras so(4) and $e(3)$ represented by differential operators.
\end{abstract}

\section{Introduction}

The commutative rings of differential operators play an important role in mathematical physics. The problem of their description, in the case of one independent variable, was set up and solved in the works by Schur [1] and Burchnall-Chaundy [2]. Later on, these results have found numerous applications in the theory of integrable equations of the Korteweg-de Vries type; we mention only the description of finite-gap operators obtained by Novikov, Krichever and others [3, 4], and the algorithm of checking necessary integrability conditions developed by Shabat and others [5].

In the case of two independent variable, the generalizations in different directions are possible. In general, the problem on a pair of commuting operators $[\hat{H}, \hat{K}]=0$ is not very well posed and the question about additional assumptions arises. Apart from the conditions suggested by the logic of the problem itself (for instance, commutativity of a triple of operators), it is also natural to select the classes of operators which are important in applications. Here we consider one such class related to the quantum tops on $s o(4)$ and $e(3)$. The corresponding operator $\hat{H}$ is of a very special form

$$
\hat{H}=a(x) D_{x}^{2}+2 f(x, y) D_{x} D_{y}+b(y) D_{y}^{2}+\ldots
$$

where $a, b$ are fourth degree polynomials, $f(x, y)$ is a biquadratic polynomial and dots denote the lower order terms, also with special coefficients. This form appears if we consider a Hamiltonian which is quadratic with respect to the generators of Lie algebra, for instance

$$
\hat{H}=(\hat{U}, A \hat{U})+(\hat{V}, B \hat{V})+2(\hat{U}, F \hat{V})+(\vec{c}, \hat{U})+(\vec{d}, \hat{V})
$$


in the case of $s o(4)$, and use a differential representation such that generators $U, V$ are replaced with differential operators of first order with quadratic coefficients (see equations (6), (8) below). The order of operator $\hat{K}$ does not exceed 4 in the known examples and, respectively, the degree of its coefficients does not exceed 8 . The original classical version of the problem deals with Hamiltonians polynomial in momenta which are in involution with respect to Poisson-Darboux bracket, instead of commuting differential operators. The conditions of "commutativity of the principal part" [6], that is, cancellation of the leading terms in the commutator, coincide for the classical and quantum cases. In more details, this relation is illustrated by the diagram

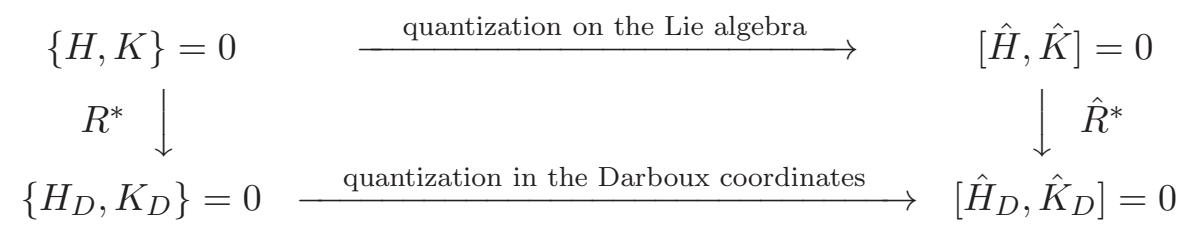

Here $R$ denotes a representation of the Lie-Poisson bracket in the Darboux coordinates, and $H_{D}$ is the image of the Hamiltonian $H$ under the pullback map, that is $H_{D}=R^{*}(H)=H \circ R$. Analogously, $\hat{R}$ denotes a representation of the Lie algebra of differential operators and $\hat{H}_{D}=\hat{R}^{*}(\hat{H})=\hat{H} \circ \hat{R}$. The horizontal arrows correspond to the passage from the Lie-Poisson bracket to the commutator on the Lie algebra and from the Poisson-Darboux bracket to the Heisenberg algebra

$$
\left\{p_{i}, x_{i}\right\}=\delta_{i j} \quad \rightarrow \quad\left[D_{x_{i}}, x_{j}\right]=\delta_{i j} .
$$

This passage, on both levels, is performed by choosing a suitable ordering of the monomials of Hamiltonians $H, K$, which is equivalent to adding the lower order terms ("quantum corrections").

Let us remind that the description of the classical tops admitting an additional first integral is still an open problem. In the so(4) case, the necessary condition were derived by Veselov [7] (see [8] for a more precise result) which define a certain varieties in the parameter space. However, the number of parameters in the known examples is less at least by 1 than the dimension of these varieties. Some classification results are presented in paper [9]. Is it possible to make progress in this problem, or, more precisely, in its quantum version, by use of the language of differential operators? As a first step, in this paper we construct the differential operators which correspond to several most important examples. In particular, these examples suggest that probably the simplest necessary condition of integrability is that the discriminant of the leading part of $\hat{H}$ is factorable:

$$
f(x, y)^{2}-a(x) b(y)=w(x, y) \tilde{w}(x, y)
$$


where $w, \tilde{w}$ are, as well as $f$, biquadratic polynomials. Notice that this relation is important in the problem of separation of variables [10]. It is not clear now, how to prove this property in the general setting, using only the assumption that there exists a commuting operator $\hat{K}$ of arbitrary order. From the computational point of view, the advantages of the differential representation is that it takes care of such issues as ordering in the operator algebra and Casimir operators. Notice that representation $\hat{R}$ is defined on a fixed level set of Casimir operators and their values occur in $\hat{H}_{D}, \hat{K}_{D}$ as independent parameters (their number is equal to the rank of Lie algebra, that is 2 in $s o(4)$ and $e(3)$ cases $)$. Splitting of the commutation relation with respect to these parameters makes its analysis more easy.

Contents of the paper are the following. The so(4) case considered in section 2 includes the tops of: Schottky-Manakov [11, 12], Stekloff [13], Adlervan Moerbeke [14, 8] and Sokolov [15]. The quantization of the SchottkyManakov top was studied in paper [16]. The cases of Adler-van Moerbeke and Sokolov are more complicated (the operator $\hat{K}$ is of fourth order) and, up to our knowledge, their quantization is obtained here for the first time.

In section 3, we consider tops on $e(3)$ : the Clebsch top [17], the Kowalevskaya top [18] (quantization is due to Laporte [19]), the Kowalevskaya gyrostat and the Goryachev-Chaplygin case. The quantization of models on $e(3)$ is rather well studied by Komarov and others [20, 21, 22, 23, 24].

Certainly, the above examples do not exhaust all known cases. An account of the known results and a detailed bibliography can be found in book [25]. Other directions in the problem on commuting differential operators were developed by many authors, we mention only papers [26, 27].

Notations. We denote $x=x_{1}, y=x_{2}$ for short. The definitions of Poisson brackets and commutators do no include vanishing brackets. The subscripts $i, j, k$ always denote an arbitrary permutation of $1,2,3$. The imaginary unit is denoted as $\mathbf{i}$. The notations

$$
\vec{a}=\left(a_{1}, a_{2}, a_{3}\right), \quad(\vec{a}, \vec{b})=a_{1} b_{1}+a_{2} b_{2}+a_{3} b_{3}
$$

are used. The following polynomials are often used in writing a compact form of Hamiltonians in Darboux coordinates:

$$
\begin{gathered}
W(a, b, c ; x, y)=a\left(x^{2}-1\right)\left(y^{2}-1\right)-b\left(x^{2}+1\right)\left(y^{2}+1\right)+4 c x y, \\
R(a, b, c ; x)=W(a, b, c, x, x)=(a-b)\left(x^{4}+1\right)+2(2 c-a-b) x^{2} .
\end{gathered}
$$

Notice that operator $W(a, b, c ; x, y) D_{x} D_{y}$ is form invariant under the inversion $x \rightarrow 1 / x, y \rightarrow 1 / y$. 


\section{Tops on $s o(4) \simeq s o(3) \oplus s o(3)$}

\subsection{Darboux coordinates and operator representation}

The Lie-Poisson bracket on so(3) and its Casimir function are defined by equations

$$
\left\{U_{i}, U_{j}\right\}=\mathbf{i} \varepsilon_{i j k} U_{k}, \quad(U, U)=s_{1}^{2}
$$

where $U$ denotes the vector $\left(U_{1}, U_{2}, U_{3}\right)$. A representation in Darboux coordinates is given by relations

$$
\begin{gathered}
U_{1}=-\frac{1}{2}\left(x^{2}-1\right) p_{1}+s_{1} x, \quad U_{2}=-\frac{\mathbf{i}}{2}\left(x^{2}+1\right) p_{1}+\mathbf{i} s_{1} x, \\
U_{3}=-x p_{1}+s_{1} .
\end{gathered}
$$

In order to quantize, we pass to the operator algebra

$$
\left[\hat{U}_{i}, \hat{U}_{j}\right]=\mathbf{i} \varepsilon_{i j k} \hat{U}_{k}, \quad(\hat{U}, \hat{U})=j_{1}\left(j_{1}+1\right)
$$

which is represented by differential operators as follows:

$$
\begin{gathered}
\hat{U}_{1}=-\frac{1}{2}\left(x^{2}-1\right) D_{x}+j_{1} x, \quad \hat{U}_{2}=-\frac{\mathbf{i}}{2}\left(x^{2}+1\right) D_{x}+\mathbf{i} j_{1} x, \\
\hat{U}_{3}=-x D_{x}+j_{1} .
\end{gathered}
$$

The Lie algebra $s o(4) \simeq s o(3) \oplus s o(3)$ is represented as the direct sum with a double under the following renaming:

$$
U \rightarrow V, \quad x \rightarrow y, \quad p_{1} \rightarrow p_{2}, \quad s_{1} \rightarrow s_{2}, \quad \hat{U} \rightarrow \hat{V}, \quad D_{x} \rightarrow D_{y}, \quad j_{1} \rightarrow j_{2} .
$$

The tops on so(4) are defined by Hamiltonians of the general form

$$
\hat{H}=(U, A U)+(V, B V)+(U, F V)+(\vec{c}, U)+(\vec{d}, V) .
$$

The matrices $A$ and $B$ can be chosen diagonal without loss of generality. The use of representation (6) yields a differential operator of the special form

$$
\begin{aligned}
\hat{H}_{D}= & a(x) D_{x}^{2}+f(x, y) D_{x} D_{y}+b(y) D_{y}^{2} \\
& -\left(\frac{2 j_{1}-1}{2} a^{\prime}(x)+2 c(x)+j_{2} f_{y}(x, y)\right) D_{x} \\
& -\left(\frac{2 j_{2}-1}{2} b^{\prime}(y)+2 d(y)+j_{1} f_{x}(x, y)\right) D_{y} \\
& +\frac{j_{1}\left(2 j_{1}-1\right)}{6} a^{\prime \prime}(x)+\frac{j_{2}\left(2 j_{2}-1\right)}{6} b^{\prime \prime}(y) \\
& +2 j_{1} c^{\prime}(x)+2 j_{2} d^{\prime}(y)+j_{1} j_{2} f_{x y}(x, y)+\kappa
\end{aligned}
$$


where $\kappa$ is an arbitrary constant and

$$
\begin{gathered}
a(x)=a_{0}\left(x^{4}+1\right)+a_{2} x^{2}, \quad b(y)=b_{0}\left(y^{4}+1\right)+b_{2} y^{2}, \\
c(x)=c_{2} x^{2}+c_{1} x+c_{0}, \quad d(y)=d_{2} y^{2}+d_{1} y+d_{0}, \\
f(x, y)=f_{22} x^{2} y^{2}+\cdots+f_{00} .
\end{gathered}
$$

The correspondence between (8) and (7) is given by relations

$$
\begin{gathered}
A=\operatorname{diag}\left(2 a_{0},-2 a_{0}, a_{2}\right)+\alpha I, \quad B=\operatorname{diag}\left(2 b_{0},-2 b_{0}, b_{2}\right)+\beta I, \\
\vec{c}=2\left(c_{2}-c_{0},-\mathbf{i}\left(c_{2}+c_{0}\right), c_{1}\right), \quad \vec{d}=2\left(d_{2}-d_{0},-\mathbf{i}\left(d_{2}+d_{0}\right), d_{1}\right), \\
F=\left(\begin{array}{ccc}
f_{00}-f_{02}-f_{20}+f_{22} & \mathbf{i}\left(f_{00}+f_{02}-f_{20}-f_{22}\right. & f_{21}-f_{01} \\
\mathbf{i}\left(f_{00}-f_{02}+f_{20}-f_{22}\right) & -f_{00}-f_{02}-f_{20}-f_{22} & -\mathbf{i}\left(f_{21}+f_{01}\right) \\
f_{12}-f_{10} & -\mathbf{i}\left(f_{12}+f_{10}\right) & f_{11}
\end{array}\right), \\
3 \kappa=\left(3 \alpha+a_{2}\right) j_{1}\left(j_{1}+1\right)+\left(3 \beta+b_{2}\right) j_{2}\left(j_{2}+1\right)
\end{gathered}
$$

with arbitrary constants $\alpha, \beta$.

The following remark is useful for computing spectra. It is about the relation between differential representation (6) and the matrix representation $[28$, p. 88$]$

$$
\begin{aligned}
& \hat{U}_{1} \pm \mathbf{i} \hat{U}_{2}=\hat{U}_{ \pm}, \quad \hat{U}_{3}|m, j\rangle=m|m, j\rangle, \\
& \hat{U}_{+}|m, j\rangle=\sqrt{(j-m)(j+m+1)}|m+1, j\rangle, \\
& \hat{U}_{-}|m, j\rangle=\sqrt{(j+m)(j-m+1)}|m-1, j\rangle .
\end{aligned}
$$

In the physical case we consider an orbit $j=$ const such that $j$ and $m$ are simultaneously integer or half-integer and $m$ takes the values from $-j$ to $j$, which corresponds to the matrices of the finite size $(2 j+1) \times(2 j+1)$. Formally, one can waive these restrictions and consider the infinite tridiagonal matrices which are interpreted as the difference operators

$$
a(m) T_{m}+b(m)+c(m) T_{m}^{-1}
$$

where $T_{m}: m \mapsto m+1$ is the shift operator (the matrix with units on the subdiagonal). A conjugation $\hat{A} \rightarrow f^{-1} \hat{A} f$ allows to get rid of square roots, and the representation of $s o(3)$ by shift operators appears:

$$
\begin{aligned}
& \hat{U}_{1}=-\frac{1}{4}(m-j)(m+j+1) T_{m}+T_{m}^{-1}, \\
& \hat{U}_{2}=-\mathbf{i}\left(\frac{1}{4}(m-j)(m+j+1) T_{m}+T_{m}^{-1}\right), \\
& \hat{U}_{3}=m .
\end{aligned}
$$

Another difference representation can be obtained directly from (6) via the change ("Fourier transformation")

$$
D_{x} \rightarrow m T_{m}, \quad x \rightarrow T_{m}^{-1}
$$


which preserves the Heisenberg algebra:

$$
\left[D_{x}, x\right]=1, \quad\left[m T_{m}, T_{m}^{-1}\right]=1,
$$

and yields the operators

$$
\begin{aligned}
& \hat{U}_{1}=\frac{m}{2} T_{m}+\left(j+1-\frac{m}{2}\right) T_{m}^{-1}, \\
& \hat{U}_{2}=-\mathbf{i} \frac{m}{2} T_{m}+\mathbf{i}\left(j+1-\frac{m}{2}\right) T_{m}^{-1}, \\
& \hat{U}_{3}=j-m+1 .
\end{aligned}
$$

One can check that representations (10) and (11) are equivalent up to a certain linear transformation from $S O(3)$ and the conjugation by operator $g(m) T^{-j-1}$ where function $g$ satisfies relation $g(m+2) / g(m+1)=2 j-m$.

\subsection{Schottky-Manakov top}

The commuting operators are of the form

$$
\begin{gathered}
\hat{H}=-\alpha_{1}^{2} \hat{U}_{1}^{2}-\alpha_{2}^{2} \hat{U}_{2}^{2}-\alpha_{3}^{2} \hat{U}_{3}^{2}-\alpha_{1}^{2} \hat{V}_{1}^{2}-\alpha_{2}^{2} \hat{V}_{2}^{2}-\alpha_{3}^{2} \hat{V}_{3}^{2} \\
+2 \alpha_{2} \alpha_{3} \hat{U}_{1} \hat{V}_{1}+2 \alpha_{3} \alpha_{1} \hat{U}_{2} \hat{V}_{2}+2 \alpha_{1} \alpha_{2} \hat{U}_{3} \hat{V}_{3}, \\
\hat{K}=\alpha_{1} \hat{U}_{1} \hat{V}_{1}+\alpha_{2} \hat{U}_{2} \hat{V}_{2}+\alpha_{3} \hat{U}_{3} \hat{V}_{3} .
\end{gathered}
$$

These can be obtained from the classical Hamiltonians in involution by the simple exchange $U \rightarrow \hat{U}, V \rightarrow \hat{V}$, because the problem of normal ordering does not appear in this example (each monomial contains commuting variables only). In contrast to some other examples (Stekloff, Kowalevskaya tops), no linear terms can be added to the Hamiltonians, both in classical and quantum cases, as one can prove by a direct computation with indeterminate coefficients. Applying map (6) yields the following pair of commuting differential operators:

$$
\begin{aligned}
& 4 \hat{H}_{D}=r(x) D_{x}^{2}+2 z D_{x} D_{y}+r(y) D_{y}^{2} \\
& \quad-\left(\frac{2 j_{1}-1}{2} r^{\prime}(x)+2 j_{2} z_{y}\right) D_{x}-\left(\frac{2 j_{2}-1}{2} r^{\prime}(y)+2 j_{1} z_{x}\right) D_{y} \\
& \quad+2 j_{1} j_{2} z_{x y}+\frac{j_{1}\left(2 j_{1}-1\right)}{6} r^{\prime \prime}(x)+\frac{j_{2}\left(2 j_{2}-1\right)}{6} r^{\prime \prime}(y) \\
& \quad-\frac{4}{3}\left(j_{1}\left(j_{1}+1\right)+j_{2}\left(j_{2}+1\right)\right)\left(\alpha_{1}^{2}+\alpha_{2}^{2}+\alpha_{3}^{2}\right), \\
& 4 \hat{K}_{D}=w D_{x} D_{y}-j_{2} w_{y} D_{x}-j_{1} w_{x} D_{y}+j_{1} j_{2} w_{x y}
\end{aligned}
$$

where, using the notations (2),

$$
\begin{aligned}
& r(x)=-R\left(\alpha_{1}^{2}, \alpha_{2}^{2}, \alpha_{3}^{2} ; x\right), \\
& z=z(x, y)=W\left(\alpha_{2} \alpha_{3}, \alpha_{3} \alpha_{1}, \alpha_{1} \alpha_{2} ; x, y\right), \\
& w=w(x, y)=W\left(\alpha_{1}, \alpha_{2}, \alpha_{3} ; x, y\right) .
\end{aligned}
$$


The constant in the last line of (14) ( $\kappa$ in equation (8)) has no effect on the commutativity and determines the energy shift of the ground state of the system.

Alternatively, operators (14), (15) can be obtained by another route along the diagram from Introduction. First, one should pass to the Darboux coordinates in the classical Hamiltonians $H, K$, accordingly to formulae (4). This yields

$$
\begin{aligned}
& 4 H_{D}=r(x) p_{1}^{2}+2 z p_{1} p_{2}+r(y) p_{2}^{2} \\
& \quad-\left(s_{1} r^{\prime}(x)+2 s_{2} z_{y}\right) p_{1}-\left(s_{2} r^{\prime}(y)+2 s_{1} z_{x}\right) p_{2} \\
& \quad+2 s_{1} s_{2} z_{x y}+\frac{s_{1}^{2}}{3} r^{\prime \prime}(x)+\frac{s_{2}^{2}}{3} r^{\prime \prime}(y)-\frac{4}{3}\left(s_{1}^{2}+s_{2}^{2}\right)\left(\alpha_{1}^{2}+\alpha_{2}^{2}+\alpha_{3}^{2}\right), \\
& 4 K_{D}=w p_{1} p_{2}-s_{2} w_{y} p_{1}-s_{1} w_{x} p_{2}+s_{1} s_{2} w_{x y}
\end{aligned}
$$

where $r, z, w$ are the same as above. Next, $p_{i}$ are replaced by $D_{x_{i}}$ in quadratic terms and the lower order terms are taken with indeterminate coefficients (preserving the degree with respect to $x, y$ ). So, one searches for the commuting differential operators of the form

$$
\begin{gathered}
\hat{H}_{D}=r(x) D_{x}^{2}+2 z(x, y) D_{x} D_{y}+r(y) D_{y}^{2}+a(\stackrel{3}{x}, \stackrel{1}{y}) D_{x}+b(\stackrel{1}{x}, \stackrel{3}{y}) D_{y}+c(\stackrel{2}{x}, \stackrel{2}{y}), \\
\hat{K}_{D}=w(x, y) D_{x} D_{y}+f(\stackrel{2}{x}, \stackrel{1}{y}) D_{x}+g(\stackrel{1}{x}, \stackrel{2}{y}) D_{y}+h(\stackrel{1}{x}, \stackrel{1}{y})
\end{gathered}
$$

where $r, z, w$ are given, $a, b, c, f, g, h$ are polynomials with indeterminate coefficients, and numbers above arguments show the corresponding degrees. The resulting system of equations is rather bulky, but its solution (14), (15) can be easily recovered by any system of computer algebra. Thus, both methods of quantization turn out to be equivalent, as one should expect. In the situation, when $\hat{H}$ is given and $\hat{K}$ is to be found, each method has its own strong and weak points from the point of view of computation complexity. The use of the Lie algebra generators gives a simpler equations for the coefficients, but its implementation is more difficult because it deals with noncommutative variables. The language of differential representation is more flexible and universal, but the resulting system for the coefficients of the operators is slightly more cumbersome.

\subsection{Stekloff top}

The classical Hamiltonians are of the form $\left(\alpha=\alpha_{1} \alpha_{2} \alpha_{3}\right)$

$$
\begin{aligned}
& H=-\alpha^{2}\left(\frac{1}{\alpha_{1}^{2}} U_{1}^{2}+\frac{1}{\alpha_{2}^{2}} U_{2}^{2}+\frac{1}{\alpha_{3}^{2}} U_{3}^{2}\right)+2 \alpha\left(\alpha_{1} U_{1} V_{1}+\alpha_{2} U_{2} V_{2}+\alpha_{3} U_{3} V_{3}\right), \\
& K=2 \alpha\left(\frac{1}{\alpha_{1}} U_{1} V_{1}+\frac{1}{\alpha_{2}} U_{2} V_{2}+\frac{1}{\alpha_{3}} U_{3} V_{3}\right)-\alpha_{1}^{2} V_{1}^{2}-\alpha_{2}^{2} V_{2}^{2}-\alpha_{3}^{2} V_{3}^{2} .
\end{aligned}
$$


Like in the previous example, the problem of ordering does not appear and the commuting quantum Hamiltonians are obtained just by placing hats over $U, V$. It is easy to check that the following linear terms can be added to the Hamiltonians, both in the classical and quantum cases:

$$
\begin{gathered}
H \rightarrow H-\beta_{1} \alpha_{2} \alpha_{3} U_{1}-\beta_{2} \alpha_{1} \alpha_{3} U_{2}-\beta_{3} \alpha_{1} \alpha_{2} U_{3}, \\
K \rightarrow K+\beta_{1} V_{1}+\beta_{2} V_{2}+\beta_{3} V_{3},
\end{gathered}
$$

with arbitrary $\beta_{i}$. However, we assume $\beta_{i}=0$ for the sake of simplicity.

The passage to the differential operators yields

$$
\begin{aligned}
& 4 \hat{H}_{D}=r_{1} D_{x}^{2}+2 z D_{x} D_{y}-\left(\frac{2 j_{1}-1}{2} r_{1}^{\prime}+2 j_{2} z_{y}\right) D_{x}-2 j_{1} z_{x} D_{y} \\
& +2 j_{1} j_{2} z_{x y}+\frac{j_{1}\left(2 j_{1}-1\right)}{6} r_{1}^{\prime \prime}-\frac{4}{3} j_{1}\left(j_{1}+1\right)\left(\alpha_{1}^{2} \alpha_{2}^{2}+\alpha_{2}^{2} \alpha_{3}^{2}+\alpha_{3}^{2} \alpha_{1}^{2}\right), \\
& 4 \hat{K}_{D}=2 w D_{x} D_{y}+r_{2} D_{y}^{2}-2 j_{2} w_{y} D_{x}-\left(\frac{2 j_{2}-1}{2} r_{2}^{\prime}+2 j_{1} w_{x}\right) D_{y} \\
& \quad+2 j_{1} j_{2} w_{x y}+\frac{j_{2}\left(2 j_{2}-1\right)}{6} r_{2}^{\prime \prime}-\frac{4}{3} j_{2}\left(j_{2}+1\right)\left(\alpha_{1}^{2}+\alpha_{2}^{2}+\alpha_{3}^{2}\right)
\end{aligned}
$$

where

$$
\begin{aligned}
r_{1} & =r_{1}(x)=-R\left(\alpha_{2}^{2} \alpha_{3}^{2}, \alpha_{3}^{2} \alpha_{1}^{2}, \alpha_{1}^{2} \alpha_{2}^{2} ; x\right), \\
r_{2} & =r_{2}(y)=-R\left(\alpha_{1}^{2}, \alpha_{2}^{2}, \alpha_{3}^{2} ; y\right), \\
z & =z(x, y)=\alpha_{1} \alpha_{2} \alpha_{3} W\left(\alpha_{1}, \alpha_{2}, \alpha_{3} ; x, y\right), \\
w & =w(x, y)=W\left(\alpha_{2} \alpha_{3}, \alpha_{3} \alpha_{1}, \alpha_{1} \alpha_{2} ; x, y\right) .
\end{aligned}
$$

The same operators are obtained via the quantization in the Darboux variables, starting from the Hamiltonians

$$
\begin{aligned}
4 H_{D}= & r_{1} p_{1}^{2}+2 z p_{1} p_{2}-\left(s_{1} r_{1}^{\prime}+2 s_{2} z_{y}\right) p_{1}-2 s_{1} z_{x} p_{2} \\
& +2 s_{1} s_{2} z_{x y}+\frac{s_{1}^{2}}{3} r_{1}^{\prime \prime}-\frac{4}{3} s_{1}^{2}\left(\alpha_{1}^{2} \alpha_{2}^{2}+\alpha_{2}^{2} \alpha_{3}^{2}+\alpha_{3}^{2} \alpha_{1}^{2}\right), \\
4 K_{D}= & 2 w p_{1} p_{2}+r_{2} p_{2}^{2}-2 s_{2} w_{y} p_{1}-\left(s_{2} r_{2}^{\prime}+2 s_{1} w_{x}\right) p_{2} \\
& +2 s_{1} s_{2} w_{x y}+\frac{s_{2}^{2}}{3} r_{2}^{\prime \prime}-\frac{4}{3} s_{2}^{2}\left(\alpha_{1}^{2}+\alpha_{2}^{2}+\alpha_{3}^{2}\right) .
\end{aligned}
$$




\subsection{Adler-van Moerbeke top}

The classical Hamiltonians are of the following form (the parameters are related by the constraint $\left.\lambda_{1}+\lambda_{2}+\lambda_{3}=0\right)$ :

$$
\begin{aligned}
H= & \sum_{i=1}^{3}\left(-9 \lambda_{j}^{2} \lambda_{k}^{2} U_{i}^{2}+6 \lambda_{j} \lambda_{k}\left(\lambda_{j}-\lambda_{i}\right)\left(\lambda_{k}-\lambda_{i}\right) U_{i} V_{i}\right. \\
& \left.+\lambda_{j} \lambda_{k}\left(4 \lambda_{i}^{2}-\lambda_{j} \lambda_{k}\right) V_{i}^{2}\right), \\
K= & 3 \sum_{i, j} \lambda_{j}\left(\lambda_{i}-\lambda_{j}\right) U_{i} V_{i} V_{j}^{2}+\sum_{i}\left(\lambda_{i}-\lambda_{j}\right)\left(\lambda_{i}-\lambda_{k}\right) U_{i} V_{i}^{3} \\
& \quad-9 \sum_{l} U_{l}^{2} \sum_{i} \lambda_{j} \lambda_{k} U_{i} V_{i}+\frac{3}{2} \sum_{l} U_{l}^{2} \sum_{i}\left(3 \lambda_{i}^{2}-\lambda_{j}^{2}-\lambda_{k}^{2}\right) V_{i}^{2} .
\end{aligned}
$$

The quantum Hamiltonian $\hat{H}$ is obtained from $H$ by fixing hats. However, $\hat{K}$ contains monomials with noncommutative generators and the problem of ordering arises. The result of direct computation with indeterminate coefficients is the following expression for $\hat{K}$ :

$$
\begin{aligned}
\hat{K}= & \sum_{i, j} \lambda_{j}\left(\lambda_{i}-\lambda_{j}\right)\left(\hat{U}_{i} \hat{V}_{i} \hat{V}_{j}^{2}+\hat{U}_{i} \hat{V}_{j} \hat{V}_{i} \hat{V}_{j}+\hat{U}_{i} \hat{V}_{j}^{2} \hat{V}_{i}\right) \\
& +\sum_{i}\left(\lambda_{i}-\lambda_{j}\right)\left(\lambda_{i}-\lambda_{k}\right) \hat{U}_{i} \hat{V}_{i}^{3}-9\left(\sum_{l} \hat{U}_{l}^{2}+\frac{1}{3}\right) \sum_{i} \lambda_{j} \lambda_{k} \hat{U}_{i} \hat{V}_{i} \\
& +\frac{3}{2} \sum_{l} \hat{U}_{l}^{2} \sum_{i}\left(3 \lambda_{i}^{2}-\lambda_{j}^{2}-\lambda_{k}^{2}\right) \hat{V}_{i}^{2} .
\end{aligned}
$$

Thus, the quantum Hamiltonian differs from the classical one by ordering in the first sum and a quantum correction in the third sum (recall, that $\sum_{l} \hat{U}_{l}^{2}$ is the Casimir function).

The following expression for $\hat{H}_{D}$ is obtained by passage to differential operators according to general formula (8):

$$
\begin{aligned}
4 \hat{H}_{D}= & r_{1}(x) D_{x}^{2}+2 z D_{x} D_{y}+r_{2}(y) D_{y}^{2} \\
& -\left(\frac{2 j_{1}-1}{2} r_{1}^{\prime}(x)+2 j_{2} z_{y}\right) D_{x}-\left(\frac{2 j_{2}-1}{2} r_{2}^{\prime}(y)+2 j_{1} z_{x}\right) D_{y} \\
& +\frac{j_{1}\left(2 j_{1}-1\right)}{6} r_{1}^{\prime \prime}(x)+\frac{j_{2}\left(2 j_{2}-1\right)}{6} r_{2}^{\prime \prime}(y)+2 j_{1} j_{2} z_{x y} \\
& -\frac{4}{3}\left(9 j_{1}\left(j_{1}+1\right)+j_{2}\left(j_{2}+1\right)\right)\left(\lambda_{1}^{2}+\lambda_{1} \lambda_{2}+\lambda_{2}^{2}\right)^{2}
\end{aligned}
$$

where

$$
\begin{aligned}
& r_{1}(x)=-9 R\left(\lambda_{2}^{2} \lambda_{3}^{2}, \lambda_{3}^{2} \lambda_{1}^{2}, \lambda_{1}^{2} \lambda_{2}^{2}, x\right), \\
& r_{2}(x)=\frac{1}{9} r_{1}(x)+4 \lambda_{1} \lambda_{2} \lambda_{3} R\left(\lambda_{1}, \lambda_{2}, \lambda_{3}, x\right), \\
& z=z(x, y)=3 W\left(\mu_{1}, \mu_{2}, \mu_{3}, x, y\right), \quad \mu_{i}=\left(\lambda_{i}^{2}-\lambda_{j}^{2}\right)\left(\lambda_{i}^{2}-\lambda_{k}^{2}\right) .
\end{aligned}
$$


The structure of operator $\hat{K}_{D}$ is rather simple:

$$
\begin{aligned}
4 \hat{K}_{D}= & g^{2} D_{x} D_{y}^{3}+c_{1} g g_{y} D_{x} D_{y}^{2}+c_{2} g g_{x} D_{y}^{3} \\
& +\left(c_{3} g g_{y y}+c_{4} g_{y}^{2}\right) D_{x} D_{y}+\left(c_{5} g_{y} g_{x}+c_{6} g g_{x y}\right) D_{y}^{2} \\
& +\left(c_{7} g g_{y y y}+c_{8} g_{y} g_{y y}\right) D_{x}+\left(c_{9} g_{y y} g_{x}+c_{10} g_{y} g_{x y}+c_{11} g g_{x y y}\right) D_{y} \\
& +c_{12} g_{y y y} g_{x}+c_{13} g_{y y} g_{x y}+c_{14} g_{y} g_{x y y}+c_{15} g g_{x y y y},
\end{aligned}
$$

but the coefficients are cumbersome. Here $g$ denotes the polynomial

$$
g=\left(\lambda_{1}-\lambda_{2}\right)\left(x y^{3}+1\right)+3 \lambda_{3}(x+y) y
$$

related to the coefficients of $\hat{H}_{D}$ by relation

$$
z^{2}-r_{1}(x) r_{2}(y)=36 \lambda_{1} \lambda_{2} \lambda_{3}\left(\lambda_{1}-\lambda_{2}\right)\left(\lambda_{2}-\lambda_{3}\right)\left(\lambda_{3}-\lambda_{1}\right) g \tilde{g},
$$

where $\tilde{g}=\left(\lambda_{1}-\lambda_{2}\right) x(x+y)-\lambda_{3}\left(x^{3} y+1\right)$. The coefficients $c_{i}$ are, in turn, polynomials in the parameters $j_{1}, j_{2}$ :

$$
\begin{aligned}
& c_{1}=-2\left(j_{2}-1\right), \quad c_{2}=-2 j_{1}, \\
& c_{3}=\frac{1}{2}\left(3 j_{1}\left(j_{1}+1\right)+j_{2}\left(j_{2}-3\right)+2\right), \quad c_{4}=-j_{1}\left(j_{1}+1\right)+j_{2}\left(j_{2}-1\right), \\
& c_{5}=2 j_{1}\left(j_{1}+j_{2}\right), \quad c_{6}=-2 j_{1}\left(j_{1}-j_{2}+2\right), \\
& c_{7}=-\frac{j_{2}}{6}\left(9 j_{1}\left(j_{1}+1\right)-j_{2}\left(j_{2}+3\right)+4\right), \quad c_{8}=\frac{j_{2}}{2}\left(j_{1}\left(j_{1}+1\right)-j_{2}\left(j_{2}-1\right)\right), \\
& c_{9}=-\frac{j_{1}}{2}\left(3 j_{1}^{2}+4 j_{1} j_{2}+j_{2}^{2}+j_{1}+j_{2}\right), \quad c_{10}=2 j_{1}\left(j_{1}\left(j_{1}+1\right)-j_{2}\left(j_{2}-1\right)\right), \\
& c_{11}=-\frac{j_{1}}{2}\left(3 j_{1}^{2}-4 j_{1} j_{2}+j_{2}^{2}+5 j_{1}-7 j_{2}+4\right), \\
& c_{12}=\frac{j_{1} j_{2}}{6}\left(9 j_{1}^{2}+3 j_{1} j_{2}-j_{2}^{2}+6 j_{1}+1\right), \\
& c_{13}=\frac{j_{1} j_{2}}{6}\left(-3 j_{1}^{2}+5 j_{1} j_{2}+3 j_{2}^{2}-4 j_{1}+2 j_{2}-1\right), \\
& c_{14}=\frac{j_{1} j_{2}}{6}\left(-3 j_{1}^{2}-5 j_{1} j_{2}+3 j_{2}^{2}-2 j_{1}-8 j_{2}+1\right), \\
& c_{15}=\frac{j_{1} j_{2}}{6}\left(9 j_{1}^{2}-3 j_{1} j_{2}-j_{2}^{2}+12 j_{1}-6 j_{2}+7\right) .
\end{aligned}
$$

\subsection{Sokolov top}

In this example, it is convenient to use the variables

$$
m_{i}=U_{i}+V_{i}, \quad n_{i}=U_{i}-V_{i} .
$$

The Sokolov top on so(4) is defined by the Hamiltonian

$$
H=\frac{1}{2} m_{1}^{2}+\frac{1}{2} m_{2}^{2}+m_{3}^{2}+m_{3}\left(\alpha n_{1}+\beta n_{2}\right)-\frac{1}{2}\left(\alpha^{2}+\beta^{2}\right) n_{3}^{2}
$$


and additional fourth order integral is of the form

$$
\begin{aligned}
K= & m_{3}^{2}\left(2 H-m_{3}^{2}+\left(\beta m_{1}-\alpha m_{2}\right)^{2}+\left(\alpha n_{1}+\beta n_{2}\right)^{2}\right) \\
& +2 m_{3}\left(\alpha m_{1}+\beta m_{2}-\left(\alpha^{2}+\beta^{2}\right) n_{3}\right)\left(m_{1} n_{1}+m_{2} n_{2}\right) .
\end{aligned}
$$

The quantum Hamiltonian is obtained by symmetrization of noncommutative monomials:

$$
\hat{H}=\frac{1}{2} \hat{m}_{1}^{2}+\frac{1}{2} \hat{m}_{2}^{2}+\hat{m}_{3}^{2}+\left[\hat{m}_{3}, \alpha \hat{n}_{1}+\beta \hat{n}_{2}\right]^{+}-\frac{1}{2}\left(\alpha^{2}+\beta^{2}\right) \hat{n}_{3}^{2}
$$

where

$$
[a, b]^{+}=\frac{1}{2}(a b+b a) .
$$

The operator $\hat{K}$ is obtained by symmetrization as well, with some special weight coefficients. It should be noted that there exist homogeneous polynomials in the algebra $s o(4)$ which vanish identically in virtue of the commutation relations, so that the form of the operator $\hat{K}$ is not unique and one can try to simplify it by adding such polynomials. We write down one of possible versions. Let us denote

$$
\begin{aligned}
A_{a, b, c}(m, f)=a m f^{2} m+b\left[m^{2}, f^{2}\right]^{+}+c[f, m f m]^{+} & \\
& +(1-a-b-c) f m^{2} f, \\
B(m, f, g)= & m f g+g f m,
\end{aligned}
$$

then the operator

$$
\begin{aligned}
\hat{K}= & A_{\frac{1}{2}, 0, \frac{3}{4}}\left(\hat{m}_{3}, \hat{m}_{1}\right)+A_{\frac{1}{2}, 0, \frac{3}{4}}\left(\hat{m}_{3}, \hat{m}_{2}\right)-\left(\alpha^{2}+\beta^{2}\right) \hat{m}_{3}^{2} \hat{n}_{3}^{2} \\
& +A_{\frac{1}{2}, \frac{1}{4}, 1}\left(\hat{m}_{3}, \beta \hat{m}_{1}-\alpha \hat{m}_{2}\right)+A_{-\frac{3}{4},-1, \frac{7}{2}}\left(\hat{m}_{3}, \hat{m}_{3}+\alpha \hat{n}_{1}+\beta \hat{n}_{2}\right) \\
& +B\left(\hat{m}_{3}, \alpha \hat{m}_{1}+\beta \hat{m}_{2}-\left(\alpha^{2}+\beta^{2}\right) \hat{n}_{3}, \hat{m}_{1} \hat{n}_{1}+\hat{m}_{2} \hat{n}_{2}\right), \\
& -\frac{1}{2}\left[\hat{m}_{3} \hat{n}_{3},\left[\hat{m}_{3}, \alpha \hat{m}_{1}+\beta \hat{m}_{2}\right]\right]
\end{aligned}
$$

commutes with $\hat{H}$ and coincides with $K$ if all variables are commutative.

In this example, it is also convenient to modify slightly our differential representation of $s o(4)$, by changing signs $y \rightarrow-y, D_{y} \rightarrow-D_{y}$. This brings to the operators which are symmetric with respect to $x, y$. Let us denote (in general, the parameters $\alpha, \beta$ are complex)

$$
\begin{gathered}
\xi_{1}=\alpha+\mathbf{i} \beta, \quad \xi_{2}=-\alpha+\mathbf{i} \beta, \quad r(x)=x\left(\xi_{1} x+1\right)\left(x+\xi_{2}\right), \\
z=z(x, y)=\xi_{1} x y(x+y)+(x+y)^{2}+2\left(1-\xi_{1} \xi_{2}\right) x y+\xi_{2}(x+y)
\end{gathered}
$$


then $\hat{H}$ is written in the form (8) as follows:

$$
\begin{aligned}
2 \hat{H}_{D}= & r(x) D_{x}^{2}+z D_{x} D_{y}+r(y) D_{y}^{2} \\
& -\left(\frac{2 j_{1}-1}{2} r^{\prime}(x)+j_{2} z_{y}\right) D_{x}-\left(\frac{2 j_{2}-1}{2} r^{\prime}(y)+j_{1} z_{x}\right) D_{y} \\
& +\frac{j_{1}\left(2 j_{1}-1\right)}{6} r^{\prime \prime}(x)+\frac{j_{2}\left(2 j_{2}-1\right)}{6} r^{\prime \prime}(y)+j_{1} j_{2} z_{x y} \\
& +\frac{1}{3}\left(j_{1}\left(j_{1}+1\right)+j_{2}\left(j_{2}+1\right)\right)\left(\xi_{1} \xi_{2}+4\right) .
\end{aligned}
$$

Notice that the polynomial $r$ is of degree 3, because Hamiltonian (22) takes the form (7) with nondiagonal matrices $A, B$ if one returns to the variables $U, V$. The second operator is too bulky and we present explicitly only the leading terms:

$$
\begin{aligned}
\hat{K}_{D}= & w^{2}\left(x D_{x}+y D_{y}\right)^{2} D_{x} D_{y}-2 j_{2} x^{2} w w_{y} D_{x}^{3}-2 j_{1} y^{2} w w_{x} D_{y}^{3} \\
& -2 x w\left(\left(j_{1}-1\right) x w_{x}+\left(j_{1}+j_{2}-1\right) w+\left(2 j_{2}-1\right) y w_{y}\right) D_{x}^{2} D_{y} \\
& -2 y w\left(\left(2 j_{1}-1\right) x w_{x}+\left(j_{1}+j_{2}-1\right) w+\left(j_{2}-1\right) y w_{y}\right) D_{x}^{2} D_{y}+\ldots
\end{aligned}
$$

where $w=\xi_{1} x y+x+y+\xi_{2}$.

\subsection{The classical limit}

The Planck constant is introduced by simple scaling of the generators, so that commutation relations (5) are replaced with

$$
\left[\hat{U}_{i}, \hat{U}_{j}\right]=\mathbf{i} \hbar \varepsilon_{i j k} \hat{U}_{k}, \quad(\hat{U}, \hat{U})=\hbar^{2} j_{1}\left(j_{1}+1\right)
$$

and representation (6) is replaced with

$$
\begin{gathered}
\hat{U}_{1}=\hbar\left(-\frac{1}{2}\left(x^{2}-1\right) D_{x}+j_{1} x\right), \quad \hat{U}_{2}=\hbar\left(-\frac{\mathbf{i}}{2}\left(x^{2}+1\right) D_{x}+\mathbf{i} j_{1} x\right), \\
\hat{U}_{3}=\hbar\left(-x D_{x}+j_{1}\right) .
\end{gathered}
$$

The equations for the variables $V$ are changed analogously. The passage to the classical limit for any quantum operator $\hat{A}$ is defined according to the formula

$$
A=\left.\lim _{\hbar \rightarrow 0} e^{-\frac{\mathbf{i}}{\hbar}\left(p_{1} x+p_{2} y\right)}\left(\hat{A} e^{\frac{\mathbf{i}}{\hbar}\left(p_{1} x+p_{2} y\right)}\right)\right|_{j_{i}=\frac{s_{i}}{\hbar}},
$$

in particular the commutator bracket and the Casimir functions for each copy of so(3) are mapped into the Lie-Poisson bracket (3), and the formulae for the generators themselves are mapped into the Darboux coordinates representation (4).

Applying of this procedure to $\hat{H}_{D}, \hat{K}_{D}$ gives the same expressions for $H_{D}$, $K_{D}$ as the intermediate passage to the Darboux coordinates in the classical Hamiltonians $H, K$. This is guaranteed by the "correspondence principle" 
which is invariant, that is it does not depend explicitly on the choice of representation of the algebra $s o(4)$. The check of the correspondence principle is trivial for the Schottky-Manakov and Stekloff tops, because there are no quantum corrections in these systems. In the Adler-van Moerbeke case, the correct passage to the classical limit is achieved by changing one term in the expression for $\hat{K}(20)$ :

$$
\left(\sum_{l} \hat{U}_{l}^{2}+\frac{1}{3}\right) \sum_{i} \lambda_{j} \lambda_{k} \hat{U}_{i} \hat{V}_{i} \rightarrow\left(\sum_{l} \hat{U}_{l}^{2}+\frac{\hbar^{2}}{3}\right) \sum_{i} \lambda_{j} \lambda_{k} \hat{U}_{i} \hat{V}_{i}
$$

After this, all terms become homogeneous with respect to $\hbar$, and the limit $\hbar \rightarrow 0$ gives rise to the classical Hamiltonian (19).

It should be noted that the Casimir functions $\hbar^{2} j_{i}\left(j_{i}+1\right)$ are of the quantum nature, because $j_{i}$ take integer/half-integer values. The passage to the limit $\hbar \rightarrow 0$ brings to the classical (finite) quantities $s_{i}=\hbar j_{i}$. On the other hand, if we consider spins then the values $j_{i}$ are finite and therefore $s_{i} \rightarrow 0$, in accordance with a statement that the spin is a pure quantum concept.

\section{Tops on $e(3)$}

\subsection{Darboux coordinates and operator representations}

The Lie-Poisson bracket on $e(3)$ is of the form

$$
\left\{M_{i}, M_{j}\right\}=-\varepsilon_{i j k} M_{k}, \quad\left\{M_{i}, \gamma_{j}\right\}=-\varepsilon_{i j k} \gamma_{k}, \quad\left\{\gamma_{i}, \gamma_{j}\right\}=0
$$

and the Casimir functions are

$$
(M, \gamma)=l, \quad(\gamma, \gamma)=a^{2}
$$

where $M=\left(M_{1}, M_{2}, M_{3}\right), \gamma=\left(\gamma_{1}, \gamma_{2}, \gamma_{3}\right)$. We use the following representation in the Darboux coordinates:

$$
\begin{aligned}
& M_{1}=-\frac{\mathbf{i}}{2}\left(x^{2}-1\right) p_{1}-\frac{\mathbf{i}}{2}\left(y^{2}-1\right) p_{2}+\frac{l}{2 a}(x-y), \\
& M_{2}=-\frac{1}{2}\left(x^{2}+1\right) p_{1}-\frac{1}{2}\left(y^{2}+1\right) p_{2}-\mathbf{i} \frac{l}{2 a}(x-y), \\
& M_{3}=\mathbf{i}\left(x p_{1}+y p_{2}\right), \\
& \gamma_{1}=a \frac{1-x y}{x-y}, \quad \gamma_{2}=\mathbf{i} a \frac{1+x y}{x-y}, \quad \gamma_{3}=a \frac{x+y}{x-y} .
\end{aligned}
$$


A representation with real Darboux coordinates [29] should be mentioned as well:

$$
\begin{gathered}
M_{1}=-p_{1} q_{1} q_{2}+\frac{1}{2} p_{2}\left(q_{1}^{2}-q_{2}^{2}-1\right)+\frac{l q_{1}\left(q_{1}^{2}+q_{2}^{2}+1\right)}{2 a\left(q_{1}^{2}+q_{2}^{2}\right)}, \\
M_{2}=p_{2} q_{1} q_{2}+\frac{1}{2} p_{1}\left(q_{1}^{2}-q_{2}^{2}+1\right)+\frac{l q_{2}\left(q_{1}^{2}+q_{2}^{2}+1\right)}{2 a\left(q_{1}^{2}+q_{2}^{2}\right)}, \\
M_{3}=p_{1} q_{2}-p_{2} q_{1}, \\
\gamma_{1}=\frac{2 a q_{1}}{\left(q_{1}^{2}+q_{2}^{2}+1\right)}, \quad \gamma_{2}=\frac{2 a q_{2}}{\left(q_{1}^{2}+q_{2}^{2}+1\right)}, \quad \gamma_{3}=\frac{a\left(q_{1}^{2}+q_{2}^{2}-1\right)}{\left(q_{1}^{2}+q_{2}^{2}+1\right)} .
\end{gathered}
$$

Quantization replaces the Lie-Poisson bracket with the $e(3)$ commutator

$$
\left[\hat{M}_{i}, \hat{M}_{j}\right]=\mathbf{i} \varepsilon_{i j k} M_{k}, \quad\left[\hat{M}_{i}, \hat{\gamma}_{j}\right]=\mathbf{i} \varepsilon_{i j k} \gamma_{k}, \quad\left[\hat{\gamma}_{i}, \hat{\gamma}_{j}\right]=0
$$

and the Casimir operators are

$$
(\hat{\gamma}, \hat{M})=l, \quad(\hat{\gamma}, \hat{\gamma})=a^{2} .
$$

This operator algebra admits the following representation:

$$
\begin{aligned}
& \hat{M}_{1}=\frac{1}{2}\left(1-x^{2}\right) D_{x}+\frac{1}{2}\left(1-y^{2}\right) D_{y}+\frac{l}{2 a}(x-y), \\
& \hat{M}_{2}=\frac{\mathbf{i}}{2}\left(1+x^{2}\right) D_{x}+\frac{\mathbf{i}}{2}\left(1+y^{2}\right) D_{y}-\mathbf{i} \frac{l}{2 a}(x-y), \\
& \hat{M}_{3}=x D_{x}+y D_{y}, \\
& \hat{\gamma}_{1}=a \frac{1-x y}{x-y}, \quad \hat{\gamma}_{2}=\mathbf{i} a \frac{1+x y}{x-y}, \quad \hat{\gamma}_{3}=a \frac{x+y}{x-y} .
\end{aligned}
$$

Notice that all operators are invariant with respect to the change

$$
x \leftrightarrow y, \quad a \rightarrow-a .
$$

A matrix representation can be obtained by introducing the basis function $|\psi\rangle=|m, n\rangle=(x+y)^{m}(x-y)^{n}$. This choice is motivated by the denominator of generators $\gamma_{i}$ in representation (30) and the symmetry arguments. Easy computation yields $\left(M_{ \pm}=M_{1} \pm \mathbf{i} M_{2}, \gamma_{ \pm}=\gamma_{1} \pm \mathbf{i} \gamma_{2}\right)$

$$
\begin{gathered}
\hat{M}_{3}|m, n\rangle=(m+n)|m, n\rangle, \quad \hat{M}_{-}|m, n\rangle=2 m|m+1, n-1\rangle, \\
\hat{M}_{+}|m, n\rangle=-\left(n+\frac{m}{2}\right)|m+1, n\rangle-\frac{m}{2}|m-1, n+2\rangle+\frac{l}{a}|m, n+1\rangle, \\
\hat{\gamma}_{3}|m, n\rangle=a|m+1, n-1\rangle, \quad \hat{\gamma}_{-}|m, n\rangle=2 a|m, n-1\rangle, \\
\hat{\gamma}_{+}|m, n\rangle=\frac{a}{2}|m, n+1\rangle-\frac{a}{2}|m+2, n-1\rangle .
\end{gathered}
$$


In order to pass to the classical limit, the Planck constant is introduced as follows:

$$
\left[\hat{M}_{i}, \hat{M}_{j}\right]=\mathbf{i} \hbar \varepsilon_{i j k} M_{k}, \quad\left[\hat{M}_{i}, \hat{\gamma}_{j}\right]=\mathbf{i} \hbar \varepsilon_{i j k} \gamma_{k}, \quad\left[\hat{\gamma}_{i}, \hat{\gamma}_{j}\right]=0
$$

and the operator representation is replaced by equations

$$
\begin{aligned}
& \hat{M}_{1}=\hbar\left(\frac{1}{2}\left(1-x^{2}\right) D_{x}+\frac{1}{2}\left(1-y^{2}\right) D_{y}\right)+\frac{l}{2 a}(x-y), \\
& \hat{M}_{2}=\hbar\left(\frac{\mathbf{i}}{2}\left(1+x^{2}\right) D_{x}+\frac{\mathbf{i}}{2}\left(1+y^{2}\right) D_{y}\right)-\mathbf{i} \frac{l}{2 a}(x-y), \\
& \hat{M}_{3}=\hbar\left(x D_{x}+y D_{y}\right), \\
& \hat{\gamma}_{1}=a \frac{1-x y}{x-y}, \quad \hat{\gamma}_{2}=\mathbf{i} a \frac{1+x y}{x-y}, \quad \hat{\gamma}_{3}=a \frac{x+y}{x-y} .
\end{aligned}
$$

The formula for the classical limit is analogous to the so(4) case:

$$
A=\lim _{\hbar \rightarrow 0} e^{-\frac{\mathrm{i}}{\hbar}\left(p_{1} x+p_{2} y\right)}\left(\hat{A} e^{\frac{\mathrm{i}}{\hbar}\left(p_{1} x+p_{2} y\right)}\right),
$$

however, notice that here the Casimir operators (29) are pure classical. Applying this procedure to the generators $M_{i}$ yields the bracket (26). In the Kowalevskaya case this procedure results in changing of a coefficient in operator $\hat{K}(35)(\mathrm{cf}[19])$ :

$$
\hat{K}=\frac{1}{2}\left(\hat{k}_{+} \hat{k}_{-}+\hat{k}_{-} \hat{k}_{+}\right)+4 \hbar^{2}\left(\hat{M}_{1}^{2}+\hat{M}_{2}^{2}\right) .
$$

\subsection{The Clebsch top}

There is no problem of ordering in this case and the quantum top is defined by the Hamiltonians

$$
\begin{aligned}
& \hat{H}=\frac{1}{2} \sum_{i=1}^{3}\left(\hat{M}_{i}^{2}+\lambda_{i} \hat{\gamma}_{i}^{2}\right), \\
& \hat{K}=\frac{1}{2} \sum_{i=1}^{3}\left(\lambda_{i} \hat{M}_{i}^{2}-\lambda \frac{\hat{\gamma}_{i}^{2}}{\lambda_{i}}\right), \quad \lambda=\lambda_{1} \lambda_{2} \lambda_{3} .
\end{aligned}
$$


The use of representation (30) yields the following commuting differential operators:

$$
\begin{aligned}
2 \hat{H}_{D}= & -(x-y)^{2} D_{x} D_{y}+\frac{l}{a}(x-y)\left(D_{x}+D_{y}\right) \\
& +\frac{a^{2} z}{(x-y)^{2}}+a^{2}\left(\lambda_{1}+\lambda_{2}+\lambda_{3}\right), \\
8 \hat{K}_{D}= & r(x) D_{x}^{2}+2 z D_{x} D_{y}+r(y) D_{y}^{2} \\
& +\left(\frac{a-l}{2 a} r^{\prime}(x)+\frac{l}{a} z_{y}\right) D_{x}+\left(\frac{a+l}{2 a} r^{\prime}(y)-\frac{l}{a} z_{x}\right) D_{y} \\
& +\left(\lambda_{1}-\lambda_{2}\right) \frac{l^{2}}{a^{2}}(x-y)^{2}-\left(\lambda_{1}-\lambda_{2}\right) \frac{l}{a}\left(x^{2}-y^{2}\right)+a^{2} \frac{r(x) r(y)-z^{2}}{(x-y)^{4}}
\end{aligned}
$$

where

$$
r(x)=R\left(\lambda_{1}, \lambda_{2}, \lambda_{3} ; x\right), \quad z=z(x, y)=W\left(\lambda_{1}, \lambda_{2}, \lambda_{3} ; x, y\right) .
$$

Notice, that in this case identity (1) takes the form

$$
\begin{aligned}
z^{2}-r(x) r(y)=4(x-y)^{4}\left(\lambda_{1} \lambda_{2}\right. & \left.+\lambda_{2} \lambda_{3}+\lambda_{3} \lambda_{1}\right) \\
& +4(x-y)^{2} W\left(\lambda_{2} \lambda_{3}, \lambda_{3} \lambda_{1}, \lambda_{1} \lambda_{2} ; x, y\right),
\end{aligned}
$$

therefore the last term in $\hat{K}_{D}$ partially cancels. The form of classical Hamiltonians in the Darboux coordinates is analogous, with slightly different coefficients:

$$
\begin{aligned}
2 H_{D}= & -(x-y)^{2} p_{1} p_{2}+\frac{2 l}{a}(x-y)\left(p_{1}+p_{2}\right) \\
& +\frac{a^{2} z}{(x-y)^{2}}+a^{2}\left(\lambda_{1}+\lambda_{2}+\lambda_{3}\right), \\
8 K_{D}= & r(x) p_{1}^{2}+2 z p_{1} p_{2}+r(y) p_{2}^{2} \\
& -\frac{l}{a}\left(r^{\prime}(x)-2 z_{y}\right) p_{1}+\frac{l}{a}\left(r^{\prime}(y)-2 z_{x}\right) p_{2} \\
& +4\left(\lambda_{1}-\lambda_{2}\right) \frac{l^{2}}{a^{2}}(x-y)^{2}+a^{2} \frac{r(x) r(y)-z^{2}}{(x-y)^{4}} .
\end{aligned}
$$

\subsection{Kowalevskaya top}

The classical top is defined by Hamiltonians

$$
H=\frac{1}{2}\left(M_{1}^{2}+M_{2}^{2}+2 M_{3}^{2}\right)-\frac{1}{2} \gamma_{1}, \quad K=k_{+} k_{-}
$$

where

$$
k_{ \pm}=\left(M_{1} \pm \mathbf{i} M_{2}\right)^{2}+\gamma_{1} \pm \mathbf{i} \gamma_{2} .
$$


The expressions in the Darboux coordinates (26) are:

$$
\begin{gathered}
2 H=x^{2} p_{1}^{2}+\left(4 x y-x^{2}-y^{2}\right) p_{1} p_{2}+y^{2} p_{2}^{2}+\frac{2 l}{a}(x-y)\left(p_{1}+p_{2}\right)+a \frac{x y-1}{x-y}, \\
K=\left(\left(x^{2} p_{1}+y^{2} p_{2}-\frac{2 l}{a}(x-y)\right)^{2}-\frac{2 a x y}{x-y}\right)\left(\left(p_{1}+p_{2}\right)^{2}+\frac{2 a}{x-y}\right) .
\end{gathered}
$$

The quantum version of the Kowalevskaya top is of the form [19, 22]

$$
\begin{aligned}
\hat{H} & =\frac{1}{2}\left(\hat{M}_{1}^{2}+\hat{M}_{2}^{2}+2 \hat{M}_{3}^{2}\right)-\frac{1}{2} \hat{\gamma}_{1}, \\
\hat{K} & =\frac{1}{2}\left(\hat{k}_{+} \hat{k}_{-}+\hat{k}_{-} \hat{k}_{+}\right)+4\left(\hat{M}_{1}^{2}+\hat{M}_{2}^{2}\right)
\end{aligned}
$$

where

$$
\hat{k}_{ \pm}=\left(\hat{M}_{1} \pm \mathbf{i} \hat{M}_{2}\right)^{2}+\hat{\gamma}_{1} \pm \mathbf{i} \hat{\gamma}_{2} .
$$

The use of representation (30) yields the following commuting differential operators:

$$
\begin{aligned}
2 \hat{H}_{D}= & x^{2} D_{x}^{2}+\left(4 x y-x^{2}-y^{2}\right) D_{x} D_{y}+y^{2} D_{y}^{2}, \\
& +\frac{1}{a}((a+l) x-l y) D_{x}+\frac{1}{a}(l x+(a-l) y) D_{y}+a \frac{x y-1}{x-y}, \\
\hat{K}_{D}= & {\left[f^{2}-\frac{2 a x y}{x-y}, g^{2}+\frac{2 a}{x-y}\right]^{+}+4[f, g f g]^{+}-2[f g, g f]^{+}-2\left[f^{2}, g^{2}\right]^{+} }
\end{aligned}
$$

where $[a, b]^{+}=\frac{1}{2}(a b+b a)$ and

$$
f=x^{2} D_{x}+y^{2} D_{y}-\frac{l}{a}(x-y), \quad g=D_{x}+D_{y} .
$$

It is worth noticing that Hamiltonians (34), (35) admit the following generalization (Kowalevskaya gyrostat) [23]:

$$
\begin{aligned}
2 \hat{H}= & \hat{M}_{1}^{2}+\hat{M}_{2}^{2}+2 \hat{M}_{3}^{2}-\hat{\gamma}_{1}+c \hat{M}_{3}, \\
\hat{K}= & \frac{1}{2}\left(\hat{k}_{+} \hat{k}_{-}+\hat{k}_{-} \hat{k}_{+}\right)+4\left(\hat{M}_{1}^{2}+\hat{M}_{2}^{2}\right)-2 c\left(\hat{M}_{1}^{2}+\hat{M}_{2}^{2}\right) \hat{M}_{3} \\
& \quad+2 c^{2} \hat{M}_{3}^{2}+c\left(c^{2}+1\right) \hat{M}_{3}-2 c \hat{M}_{1} \hat{\gamma}_{3}-c^{2} \hat{\gamma}_{1}-\mathbf{i} c \hat{\gamma}_{2} .
\end{aligned}
$$

\subsection{Goryachev-Chaplygin case}

The quantization was considered in [21]. The Hamiltonians

$$
\begin{aligned}
& \hat{H}=\hat{M}_{1}^{2}+\hat{M}_{2}^{2}+4 \hat{M}_{3}^{2}-\hat{\gamma}_{1}+c \hat{M}_{3}, \\
& \hat{K}=4\left(\hat{M}_{1}^{2}+\hat{M}_{2}^{2}\right) \hat{M}_{3}+2 \hat{M}_{1} \hat{\gamma}_{3}-4 c \hat{M}_{3}^{2}+\left(1-c^{2}\right) \hat{M}_{3}+c \hat{\gamma}_{1}+\mathbf{i} \hat{\gamma}_{2}
\end{aligned}
$$

satisfy the relation

$$
[\hat{H}, \hat{K}]=4 \mathbf{i} l \hat{M}_{2}
$$


where $l=(\hat{\gamma}, \hat{M})$ is one of the Casimir operators on $e(3)$. Therefore, an integrable case occurs at $l=0$. The passage to operator representation (30) (at $l=0$ ) yields the commuting pair

$$
\begin{gathered}
\hat{H}_{D}=3 x^{2} D_{x}^{2}-\left(x^{2}-8 x y+y^{2}\right) D_{x} D_{y}+3 y^{2} D_{y}^{2} \\
+(c+3)\left(x D_{x}+y D_{y}\right)+a \frac{x y-1}{x-y} \\
-\hat{K}_{D}-4\left(\frac{c}{3}+1\right) \hat{H}_{D}=4 x^{3} D_{x}^{3}+4\left(x^{2}+x y+y^{2}\right)\left(x D_{x}+y D_{y}\right) D_{x} D_{y}+4 y^{3} D_{y}^{3} \\
+\left(\frac{c}{3}+3\right)\left(4(x-y)^{2} D_{x} D_{y}-(c+3)\left(x D_{x}+y D_{y}\right)\right) \\
+\frac{a}{x-y}\left((x+y)\left(\left(x^{2}-1\right) D_{x}+\left(y^{2}-1\right) D_{y}\right)-\left(\frac{c}{3}+5\right) x y+\frac{c}{3}+3\right) .
\end{gathered}
$$

\section{Spectra}

The quantization in terms of the generators of Lie algebra is universal, but the setting of a boundary value problem and computation of the spectra depend on the choice of a concrete representation. As an application, we consider here the eigenvalue problem for the Euler top on so(3) using representation (6). Recall, that quantization of this model was obtained by Kramers-Ittmann [30]. In the case of representation (6), it is natural to define the spectrum by condition that eigenfunctions are polynomial. This can be compared with Komarov-Kuznetsov paper [22] where the spectrum was found for the matrix representation, and the recent Grosset-Veselov paper [31] where the spectrum was studied for the representation in elliptic coordinates and it was shown that coefficients of the characteristic polynomial at a given level set $j=s$ are expressed through the so-called elliptic Bernoulli polynomials. The spectral problem for the tops on $s o(4)$ is rather complicated and we restrict ourselves by derivation of equations for eigenfunctions in the Schottky-Manakov case.

\subsection{Matrix representation of $s o(3)$}

Let us introduce the wave function $|m, j\rangle=x^{j-m}$, then representation (6) has the following matrix elements in this basis:

$$
\begin{gathered}
\hat{U}_{1} \pm \mathbf{i} \hat{U}_{2}=\hat{U}_{ \pm}, \quad \hat{U}_{3}|m, j\rangle=m|m, j\rangle, \\
\hat{U}_{+}|m, j\rangle=(j-m)|m+1, j\rangle, \quad \hat{U}_{-}|m, j\rangle=(j+m)|m-1, j\rangle .
\end{gathered}
$$

In this case the condition that $j-m$ is integer follows from the condition that the basis functions $x^{j-m}$ must be single-valued. If the problem admits the time-reversal symmetry $m \rightarrow-m$ then $j+m$ should be integer as well, and this implies that $j$ and $m$ are simultaneously integer or half-integer. The form of the matrix elements implies that $m=-j, \ldots, j$, and the condition $j>0$ follows from the condition that the basis function must be analytic. 
It is clear from its form that wave function on the orbit $j=$ const is a polynomial of degree $2 j$.

The wave function and the spectral problem on $s o(3)$ can be written as follows:

$$
|\psi\rangle=\sum_{m=-j}^{j} C(m, j)|m, j\rangle, \quad H|\psi\rangle=\lambda|\psi\rangle
$$

Analogously, the wave function and the spectral problem on so(4) read:

$$
|\psi\rangle=\sum_{m_{1}=-j_{1}}^{j_{1}} \sum_{m_{1}=-j_{2}}^{j_{2}} C\left(m_{1}, m_{2} ; j_{1}, j_{2}\right)\left|m_{1}, m_{2}, j_{1}, j_{2}\right\rangle, \quad H|\psi\rangle=\lambda|\psi\rangle .
$$

In the differential representation, the basis wave function on so(4) is chosen as $\left|m_{1}, m_{2}, j_{1}, j_{2}\right\rangle=x^{j_{1}-m_{1}} y^{j_{2}-m_{2}}$. Then the general wave function is a polynomial of degree $2 j_{1}$ with respect to $x$ and degree $2 j_{2}$ with respect to $y$.

\subsection{Spectrum of the Euler top on $s o(3)$}

The eigenvalue problem is of the form

$$
\hat{H} \psi_{j}^{\lambda}(x)=\lambda \psi_{j}^{\lambda}(x), \quad \hat{C} \psi_{j}^{\lambda}(x)=j(j+1) \psi_{j}^{\lambda}(x)
$$

where

$$
\hat{H}=\alpha_{1} \hat{U}_{1}^{2}+\alpha_{2} \hat{U}_{2}^{2}+\alpha_{3} \hat{U}_{3}^{2}, \quad \hat{C}=\hat{U}_{1}^{2}+\hat{U}_{2}^{2}+\hat{U}_{3}^{2} .
$$

We will assume that $\alpha_{1}+\alpha_{2}+\alpha_{3}=0$, up to an unessential shift of the spectrum.

Let us use the differential operators representation for so(3) algebra (36) and represent wave function in the form

$$
\psi_{j}^{\lambda}(x)=\sum_{k=0}^{2 j} \tilde{C}_{j}^{\lambda}(k) x^{k} .
$$

Then the eigenvalue problem is rewritten in the form of recurrent relation

$$
\begin{gathered}
\frac{1}{4}(2 j+1-k)(2 j+2-k) C_{j}^{\Lambda}(k-2) \\
+\left(\frac{1}{2}\left(j(j+1)-3(j-k)^{2}\right) \xi-\Lambda\right) C_{j}^{\Lambda}(k) \\
+\frac{1}{4}(k+1)(k+2) C_{j}^{\Lambda}(k+2)=0
\end{gathered}
$$

where $\xi=\frac{\alpha_{1}+\alpha_{2}}{\alpha_{1}-\alpha_{2}}, \Lambda=\frac{\lambda}{\alpha_{1}-\alpha_{2}}, C_{j}^{\Lambda}=\tilde{C}_{j}^{\lambda}$, with the boundary conditions on the left end

$$
C_{j}^{\Lambda}(-2)=C_{j}^{\Lambda}(-1)=0, \quad C_{j}^{\Lambda}(0)=C_{j}^{\Lambda}(1)=1 .
$$


The problem is splitting for odd and even polynomials.

If $j$ is integer then the boundary conditions on the right end is $C_{j}^{\Lambda}(2 j+$ $2)=0$ for the even polynomials and $C_{j}^{\Lambda}(2 j+1)=0$ for the odd ones.

If $j$ is half-integer then, vice-versa, the boundary conditions on the right end is $C_{j}^{\Lambda}(2 j+1)=0$ for the even polynomials and $C_{j}^{\Lambda}(2 j+2)=0$ for the odd ones.

As a result, the eigenvalues of the Hamiltonian $\hat{H}$ are zeroes of the polynomial

$$
P_{j}(\Lambda)=C_{j}^{\Lambda}(2 j+1) C_{j}^{\Lambda}(2 j+2), \quad \operatorname{deg} P_{j}(\Lambda)=2 j+1 .
$$

Let us explicitly write down several polynomials $P_{j}(\Lambda)$ normalized by the condition that the coefficient of the leading term $\Lambda^{2 j+1}$ is unit:

$$
\begin{aligned}
& P_{0}(\Lambda)=\Lambda, \\
& P_{1 / 2}(\Lambda)=\Lambda^{2}, \\
& P_{1}(\Lambda)=\frac{1}{4}(\Lambda-\xi)(2 \Lambda+\xi+1)(2 \Lambda+\xi-1), \\
& P_{3 / 2}(\Lambda)=\frac{1}{16}\left(4 \Lambda-9 \xi^{2}-3\right)^{2}, \\
& P_{2}(\Lambda)=\frac{1}{4}(\Lambda+3 \xi)(2 \Lambda-3 \xi+3)(2 \Lambda-3 \xi-3)\left(\Lambda-9 \xi^{2}-3\right)^{2}, \\
& P_{5 / 2}(\Lambda)=\left(\Lambda^{3}-7 \Lambda\left(3 \xi^{2}+1\right)+20 \xi\left(\xi^{2}-1\right)\right)^{2} .
\end{aligned}
$$

All polynomials $P_{j}(\lambda)$ with half-integer $j$ are full squares in virtue of the Kramers theorem [28, p. 225] about the double degeneration of the systems with half-integer value of the spin.

\subsection{Matrix representation for the Schottky-Manakov top}

In the Schottky-Manakov case we have two consistent eigenvalue problems $\hat{H} \psi=\lambda \psi, \hat{K} \psi=\mu \psi$. The wave function is of the form

$$
\psi_{j_{1}, j_{2}}^{\lambda, \mu}(x, y)=\sum_{k=0}^{2 j_{1}} \sum_{l=0}^{2 j_{2}} C_{j_{1}, j_{2}}^{\lambda, \mu}(k, l) x^{k} y^{l} .
$$

In the lattice representation, the 5-point equation appears for the eigenvalues $\mu$ :

$$
\begin{aligned}
& \left(\alpha_{1}-\alpha_{2}\right)\left(2 j_{1}+1-k\right)\left(2 j_{2}+1-l\right) C_{j_{1}, j_{2}}^{\lambda, \mu}(k-1, l-1) \\
+ & \left(\alpha_{1}-\alpha_{2}\right)(k+1)(l+1) C_{j_{1}, j_{2}}^{\lambda, \mu}(k+1, l+1) \\
+ & 2\left(2\left(j_{1}-k\right)\left(j_{2}-l\right) \alpha_{3}-\mu\right) C_{j_{1}, j_{2}}^{\lambda, \mu}(k, l) \\
+ & \left(\alpha_{1}+\alpha_{2}\right)\left(2 j_{1}+1-k\right)(l+1) C_{j_{1}, j_{2}}^{\lambda, \mu}(k-1, l+1) \\
+ & \left(\alpha_{1}+\alpha_{2}\right)(k+1)\left(2 j_{2}+1-l\right) C_{j_{1}, j_{2}}^{\lambda, \mu}(k+1, l-1)=0,
\end{aligned}
$$


and 9-point one for the eigenvalues $\lambda$ :

$$
\begin{aligned}
& \left(\alpha_{2}^{2}-\alpha_{1}^{2}\right)(k+1)(k+2) C_{j_{1}, j_{2}}^{\lambda, \mu}(k+2, l) \\
+ & \left(\alpha_{2}^{2}-\alpha_{1}^{2}\right)(l+1)(l+2) C_{j_{1}, j_{2}}^{\lambda, \mu}(k, l+2) \\
+ & 2 \alpha_{3}\left(\alpha_{2}-\alpha_{1}\right)(k+1)(l+1) C_{j_{1}, j_{2}}^{\lambda, \mu}(k+1, l+1) \\
+ & 2 \alpha_{3}\left(\alpha_{2}+\alpha_{1}\right)(k+1)\left(2 j_{2}+1-l\right) C_{j_{1}, j_{2}}^{\lambda, \mu}(k+1, l-1) \\
+ & \left(( \alpha _ { 1 } - \alpha _ { 2 } ) ^ { 2 } \left(\left(2 j_{1}+1-k\right)^{2}+\left(2 j_{2}+1-l\right)^{2}\right.\right. \\
& \quad-2\left(j_{1}+j_{2}+1-k\right)\left(j_{1}+j_{2}+1-l\right) \\
& \left.\quad-\left(j_{1}-j_{2}\right)^{2}-j_{1}\left(j_{1}+1\right)-j_{2}\left(j_{2}+1\right)\right) \\
& \quad-4 \alpha_{3}^{2}\left(\left(j_{1}-k\right)^{2}+\left(j_{2}-l\right)^{2}\right) \\
& \left.\quad+\left(\alpha_{2}+\alpha_{1}\right)^{2}\left(\left(j_{1}+j_{2}-k-l\right)^{2}-j_{1}\left(j_{1}+1\right)-j_{2}\left(j_{2}+1\right)\right)\right) C_{j_{1}, j_{2}}^{\lambda, \mu}(k, l) \\
+ & \left(\alpha_{2}^{2}-\alpha_{1}^{2}\right)\left(2 j_{1}+1-k\right)\left(2 j_{1}+2-k\right) C_{j_{1}, j_{2}}^{\lambda, \mu}(k-2, l) \\
+ & \left(\alpha_{2}^{2}-\alpha_{1}^{2}\right)\left(2 j_{2}+1-l\right)\left(2 j_{2}+2-l\right) C_{j_{1}, j_{2}}^{\lambda, \mu}(k, l-2) \\
+ & 2 \alpha_{3}\left(\alpha_{2}+\alpha_{1}\right)\left(2 j_{1}+1-k\right)(l+1) C_{j_{1}, j_{2}}^{\lambda, \mu}(k-1, l+1) \\
+ & 2 \alpha_{3}\left(\alpha_{2}-\alpha_{1}\right)\left(2 j_{1}+1-k\right)\left(2 j_{2}+1-l\right) C_{j_{1}, j_{2}}^{\lambda, \mu}(k-1, l-1)=0 .
\end{aligned}
$$

The boundary conditions are: $C_{j_{1}, j_{2}}^{\lambda, \mu}(k, l)=0$ if the pair $(k, l)$ lies outside the rectangle with the vertices $(0,0),\left(j_{1}, 0\right),\left(j_{1}, j_{2}\right),\left(0, j_{2}\right)$.

Solutions $C_{j_{1}, j_{2}}^{\lambda, \mu}(k, l)$ split into solutions on two sublattices: a solution is called "even" if it vanishes at odd $k+l$, and it is called "odd" if it vanishes at even $k+l$.

Actually, one can avoid solving 9-point equation: it is sufficient to determine the wave functions from the 5 -point equation and then the substitution into the 9-point one allows to determine the relation between $\lambda_{i}$ and $\mu_{i}$. A plausible answer is that the pairs $\left(\lambda_{i}, \mu_{i}\right)$ lie on a certain algebraic curve.

Consider the case $j_{1}=j, j_{2}=\frac{1}{2}$ as an example. The wave function is a superposition of odd and even ones. The even wave function is of the form

$$
\psi=C_{0}+C_{1} x y+C_{2} x^{2}+C_{3} x^{3} y+\ldots
$$

and coefficients satisfy the boundary conditions $C_{-2}=C_{-1}=C_{2 j+1}=0$ and recurrent relations

$$
\begin{aligned}
\frac{1}{2}\left(\alpha_{1}+(-1)^{k} \alpha_{2}\right)(2 j+1-k) C_{k-1} & +\left(\alpha_{3}(j-k)(-1)^{k}-\mu\right) C_{k} \\
& +\frac{1}{2}\left(\alpha_{1}-(-1)^{k} \alpha_{2}\right)(k+1) C_{k+1}=0 .
\end{aligned}
$$

Up to the constant factors, one finds

$C_{0}=0, \quad C_{1}=\mu-j \alpha_{3}, \quad C_{2}=\mu^{2}-\alpha_{3} \mu-\alpha_{3}^{2} j(j-1)-\frac{1}{2} j\left(\alpha_{1}-\alpha_{2}\right)^{2}, \quad \ldots$ 
The odd wave function is of the form

$$
\psi=B_{0} y+B_{1} x+B_{2} x^{2} y+B_{3} x^{3}+\ldots,
$$

the coefficients satisfy the boundary conditions $B_{-2}=B_{-1}=B_{2 j+1}=0$ and recurrent relations

$$
\begin{aligned}
\left(\alpha_{1}-(-1)^{k} \alpha_{2}\right)(2 j+1-k) B_{k-1}+2 & \left(\alpha_{3}(k-j)(-1)^{k}-\mu\right) B_{k} \\
+ & \left(\alpha_{1}+(-1)^{k} \alpha_{2}\right)(k+1) B_{k+1}=0 .
\end{aligned}
$$

Up to the constant factors, one finds

$$
B_{0}=0, \quad B_{1}=\mu+j \alpha_{3}, \quad B_{2}=\mu^{2}+\alpha_{3} \mu-\alpha_{3}^{2} j(j-1)-\frac{1}{2} j\left(\alpha_{1}-\alpha_{2}\right)^{2}, \quad \ldots
$$

The eigenvalues are found from equation

$$
P_{j, 1 / 2}(\mu)=C(2 j+1) B(2 j+1)=0, \quad \operatorname{deg} P_{j, 1 / 2}(\mu)=2(2 j+1) .
$$

The polynomial $P_{j, 1 / 2}(\mu)$ is a full square if $j$ is integer, in accordance with the Kramers theorem, because then $j+\frac{1}{2}$ is half-integer.

\section{Acknowledgements}

The authors thank I.M. Krichever and V.V. Sokolov for their interest to this work and useful remarks. The research was supported by grants NSh6501.2010.2 and RFBR 10-01-00088.

\section{References}

[1] I. Schur. Über vertauschbare lineare Differentialausdrücke. Sitzungsber. Berliner Math. Ges. 4 (1905) 2-8.

[2] J.L. Burchnall, T.W. Chaundy. Commutative ordinary differential operators. Proc. R. Soc. Lond. A 118 (1928) 557-583

[3] S.P. Novikov. Periodic problem for the Korteweg-de Vries equation. I. Funct. Anal. Appl. 8:3 (1974) 236-246.

[4] I.M. Krichever. Commutative rings of ordinary linear differential operators. Funct. Anal. Appl. 12:3 (1978) 175-185.

[5] A.V. Mikhailov, A.B. Shabat, R.I. Yamilov. The symmetry approach to classification of nonlinear equations. Complete lists of integrable systems. Russ. Math. Surveys 42:4 (1987) 1-63. 
[6] R.A. Gabiev, A.B. Shabat. On differential operators which commute in principal parts. Theor. Math. Phys. (2011).

[7] A.P. Veselov. On the integrability conditions for the Euler equation on so(4). Dokl. Akad. Nauk SSSR 270:6 (1983) 1298-1300.

[8] A.G. Reyman, M.A. Semenov-Tian-Shansky. A new integrable case of the motion of the 4-dimensional rigid body. Comm. in Math. Phys. 105:3 (1986) 461-472.

[9] V.V. Sokolov. One class of quadratic so(4) Hamiltonians. Doklady Math. 69:1 (2004) 108-111.

[10] V.G. Marikhin, V.V. Sokolov. Separation of variables on a nonhyperelliptic curve. Reg. and Chaot. Dyn. 10:1 (2005) 59-70.

[11] F. Schottky. Über das analytische Problem der Rotation eines starren Körpers in Raume von vier Dimensionen. Sitzungsberichte der Königlich preussischen Academie der Wissenschaften zu Berlin XIII (1891) 227232.

[12] S.V. Manakov. Note on the integration of Euler's equations of the dynamics of an $n$-dimensional rigid body. Funct. Anal. Appl. 10:4 (1976) 328-329.

[13] V.A. Stekloff. Sur le mouvement d'un corps solide ayant une cavite de forme ellipsoidale remple par un liquide incompressible en sur les variations des latitudes. Ann. de la fac. des Sci. de Toulouse, Ser. 3, v. 1 (1909).

[14] M. Adler, P. van Moerbeke. A new geodesic flow on $S O(4)$. Probability, statistical mechanics and number theory. Adv. Math. Suppl. Stud 9 (1986) 81-96.

[15] V.V. Sokolov. Generalized Kowalewski top: new integrable cases on $e(3)$ and so(4). In: "Kowalevski property", ed. V.B. Kuznetsov, CRM Proceedings and Lecture Notes, 32 (2002) 307-313. ArXiV: nlin.SI/0110022, 2001.

[16] I.V. Komarov, V.B. Kuznetsov. Quantum Euler-Manakov top on the three-sphere $S_{3}$. J. Phys. A 24:13 (1991) L737-742.

[17] A. Clebsch. Über die Bewegung eines Körpers in einer Flüssigkeit. Math. Annalen 3 (1870) 238-262.

[18] S.V. Kowalevski. Sur le probleme de la rotation d'un corps solide autour d'un point fixe. Acta Math. 12:1 (1889) 177-232. 
[19] O. Laporte. Note on Kowalewski's top in quantum mechanics. Phys. Rev. 43 (1933) 548-551.

[20] I.V. Komarov. Kowalewski basis for the hydrogen atom. Theor. Math. Phys. 41:1 (1981) 320-324.

[21] I.V. Komarov. Goryachev-Chaplygin top in quantum mechanics. Theor. Math. Phys. 50:3 (1982) 265-270.

[22] I.V. Komarov, V.B. Kuznetsov. Semiclassical quantization of Kowalewski top. Theor. Math. Phys. 73:3 (1987) 1255-1263.

[23] I.V. Komarov. Remarks on Kowalevski's top. J. Phys. A 34 (2001) 2111-2120.

[24] A. Ramani, B. Grammaticos, B. Dorizzi. On the quantization of the Kowalevskaya top. Phys. Lett. A 101:2 (1984) 69-71.

[25] A.V. Borisov, I.S Mamaev. Rigid body dynamics - Hamiltonian methods, integrability, chaos. Moscow-Izhevsk: Institute of Computer Science, 2005, 576 p. [in Russian].

[26] J. Hietarinta. Pure quantum integrability. Phys. Lett. A 246 (1998) 97-104.

[27] M.A. Olshanetsky, A.M. Perelomov. Quantum integrable systems related to Lie algebras. Phys. Reports 94:6 (1983) 313-404.

[28] L.D. Landau, E.M. Lifshitz. Course of theoretical physics. vol. 3. Quantum Mechanics. Nonrelativistic theory. 3rd edition, Oxford, Pergamon Press, 1977.

[29] V.G. Marikhin, V.V. Sokolov. Transformation of a pair of commuting Hamiltonians quadratic in momenta to a canonical form and on a partial real separation of variables for the Clebsch top. Regul. Chaotic Dyn. 15:6 (2010) 652-658.

[30] H.A. Kramers, G.P. Ittmann. Zur Quantelung des asymmetrischen Kreisels. Z. Physik 53 (1929) 553-565; 58 (1929) 217-231; 60 (1930) 663-681.

[31] M-P. Grosset, A.P. Veselov. Lamé equation, quantum top and elliptic Bernoulli polynomials. Proc. Edinburgh Math. Soc. (Series 2) 51 (2008) 635-650. 